
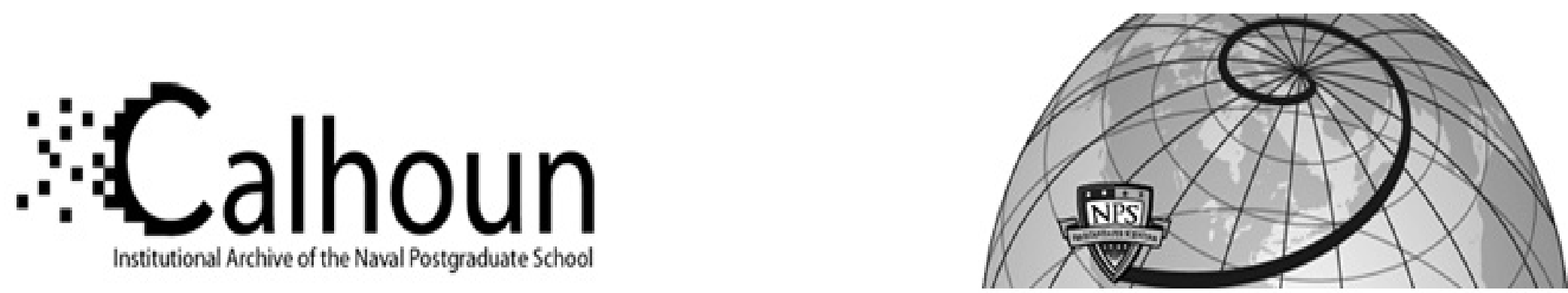

Calhoun: The NPS Institutional Archive DSpace Repository

Optimal Control of Shock Wave Attenuation using Liquid Water Droplets with Application to Ignition Overpressure in Launch Vehicles

Moshman, Nathan D.; Sritharan, Sivaguru S.; Hobson, Garth V.

International Journal of Flow Control, Volume 3, Number 4, 2011

https://hdl.handle.net/10945/44960

This publication is a work of the U.S. Government as defined in Title 17, United States Code, Section 101. Copyright protection is not available for this work in the United States.

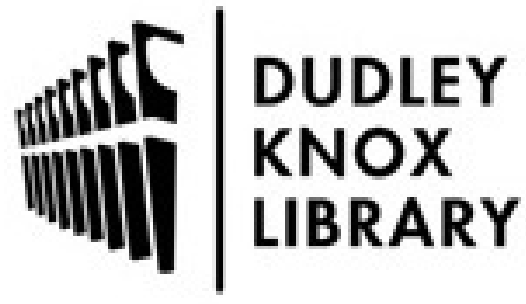

http://www.nps.edu/library
Calhoun is the Naval Postgraduate School's public access digital repository for research materials and institutional publications created by the NPS community. Calhoun is named for Professor of Mathematics Guy K. Calhoun, NPS's first appointed -- and published -- scholarly author.

Dudley Knox Library / Naval Postgraduate School 411 Dyer Road / 1 University Circle Monterey, California USA 93943 


\title{
Optimal Control of Shock Wave Attenuation using Liquid Water Droplets with Application to Ignition Overpressure in Launch Vehicles
}

\author{
Nathan D. Moshman, Sivaguru S. Sritharan and Garth V. Hobson \\ 700 Dyer Road Monterey, CA 93943
}

\begin{abstract}
This paper presents the first solution of an optimal control problem concerning unsteady blast wave attenuation where the control takes the form of the initial distribution of liquid water droplets. An appropriate two-phase flow model is adopted for compressible homogeneous two-phase flows. The dynamical system includes an empirical model for water droplet vaporization, the dominant mechanism for attenuating the jump in pressure across the shock front. At the end of the simulation interval, an appropriate target state is defined such that the jump in pressure of the target state is less than that of the simulated blast wave. Given the nature of the non-linear system, the final time must also be a free variable. A novel control algorithm is presented which can satisfy all necessary conditions of the optimal system and avoid taking a variation at the shock front. The adjoint-based method is applied to NASA's problem of Ignition Overpressure blast waves generated during ignition of solid grain rocket segments on launch vehicles. Results are shown for a range of blast waves that are plausible to see in the launch environment of the shuttle. Significant parameters of effective droplet distributions are identified.
\end{abstract}

\section{INTRODUCTION}

There exists a wide range of multi-phase and multi-fluid flow regimes. Much care must be taken in selecting an appropriate model and numeric method. Presently, the flow of interest consists of a gaseous carrier phase with many suspended water droplets. These two fluids' parameters are drastically different and the vast majority of numeric methods in the literature are ill-adapted since they introduce artificial diffusion and mixing at interfaces [1-3]. Other methods are poorly suited to mixtures where thermodynamic equilibrium cannot be reasonably assumed except at interfaces. Since the water droplets of interest will be smaller than $1 \mathrm{~mm}$, their properties can be assumed to be homogeneous between computational cells and, therefore, many interfaces will be present in each cell. It will be impractical to use interface tracking methods or solve separate sets of equations for each fluid and to couple their properties at the interface. Instead, the following calculations implement a method proposed by [4] which has the advantage of solving the same set of differential equations throughout the domain. The method uses different equations of state for each phase and allows a difference in temperature at the gas-liquid interfaces. To close the system, a volume fraction variable $\alpha$ is introduced and a non-conservative PDE is appended to the conservative system. The method assumes that both phases are compressible which yields a hyperbolic system.

Ignition overpressure (IOP) is a phenomenon present at the start of an ignition sequence in launch vehicles using solid-grain propellants. When the grain is ignited the pressure inside the combustion chamber quickly rises several orders of magnitude. This drives hot combustion products toward the nozzle and out to the open atmosphere at supersonic speeds. An IOP wave is an unsteady shock wave which originates from the exit plane of the nozzle and propagates spherically outward. From previous launch data [5-7] it is known that overpressures that the body of the rocket experiences are of the order $2: 1$. The region below the nozzle, such as the launch pad trench, will experience further compression due to displacement of gas along the blast wave's direction of propagation and overpressures can be as high as 10:1. The portions of the IOP wave that become incident on the rocket body or launch platform components must have an overpressure below a known threshold to avoid costly damage and enable a long operational lifetime. This is the purpose of the water suppression system which is integrated into 

Application to Ignition Overpressure in Launch Vehicles

the launch platform. The current technique used by NASA and other launch providers is to spray water into the region around the nozzle before ignition. This forces the IOP wave to propagate through water before becoming incident on the rocket body or platform components. Through several dissipative mechanisms this causes a sufficient decrease to the pressure jump across the shock, mitigating damage.

A survey of empirical data in the literature was carried out [8-15] to understand how droplets can be used as a control action in two-phase flow. With blast mitigation as the process of interest, Ananth et al. [13] identified several desirable and undesirable effects. Drag on the droplets by the surrounding gas dissipates momentum. As droplets breakup, the gas does work against the surface tension which dissipates energy from the gas. Droplets absorb sensible, latent and radiative heat which further transfers energy from the gas. Since water vapor has a higher heat capacity than air, after water changes phase from liquid to gas the resulting gas mixture can further absorb heat from the gas. An undesirable effect occurs when liquid turns to water vapor so rapidly that the gas mixture density increase dominates the effect on pressure according to the constitutive relation. The relative importance of each interaction mechanism isn't fully understood and will change depending on the flow regimes.

The flow regime of interest will have shocks present with pressure ratios $(2: 1-10: 1)$ and temperatures up to several thousand Kelvin. Practical droplet atomizers can make droplets with diameters $D_{p}$ between $25 \mu \mathrm{m}$ and $500 \mu \mathrm{m}$; any larger and the assumption of homogenized droplet distributions within computational cells will be begin to break down. A shock incident on a cloud of water droplets in this size range will pass through them so quickly that the droplets will not appear to react until after the shock front is spatially isolated downstream. Droplets will start to get dragged along in the direction of the gas flow, breakup and vaporize. If droplets are large, $D_{p} \geq 100 \mu \mathrm{m}$, then in the presence of strong shocks they catastrophically breakup into many small droplets $D_{p} \leq 25 \mu \mathrm{m}$. Droplet breakup ceases below a Weber number of 12 , when inertial forces dominate over surface tension $[11,15]$. Shock attenuation will be greatest when the droplets can sink the most energy out of the gas in a given interval of space. This will cause the greatest decrease in pressure to the driving gas behind the shock. Pressure information will travel at the local sound speed toward the shock front and ultimately cause a decrease in pressure equal to the diminished driver gas pressure.

There are general trends in the data which help categorize the relative importance of all of the dissipative mechanisms present. First and foremost, droplet vaporization can extract orders of magnitude more energy from the gas than droplet breakup can. Secondly, the latent heat of vaporization is the most significant dissipative mechanism [13]. Lastly, it has been empirically demonstrated [15] that a non-dimensional droplet-flow parameter can predict overpressure attenuation level. The experiment showed that a nondimensional flow parameter directly related to exposed droplet surface area could be correlated to the greater decrease in overpressure as measured at a fixed location downstream. Shock tube experiments were conducted which varied the droplet size and shock strength. The data suggests that maximizing the exposed surface area of the droplets will yield the greatest enhancement of IOP attenuation.

\subsection{Recent Research on IOP Attenuation}

For many years, the water injection strategy for handling the shuttle's IOP transient blast has been based on order-of-magnitude estimates relating the amount of energy dissipation required to the total amount of water used [16]. More than enough water was used and the results were sufficient. For the heavy lift vehicles of the future, IOP blast waves will be more substantial and require a better understanding of how the water affects the IOP strength. More recently, CFD research is underway at NASA [17, 18] and in the private sector to predict, with greater precision, the launch environment during ignition for various solid grain rockets $[19,20]$.

The first work on optimizing one of these precise CFD simulations was a parametric study that looked at water arrangement in the nozzle region and how it affected the maximum IOP strength. At NASA Huntsville, Canabal showed in his dissertation, [21], and a later publication, [22], that attenuation is very insensitive to droplet velocity and demonstrated the existence of an optimal water injection arrangement. Water cooling the plume near the nozzle has the greatest desirable effect of attenuating the transmitted IOP strength. However, an excessive amount of water near the nozzle causes obstruction to the blast wave and intensifies pressure. The results suggest an optimal arrangement of water exists but there are still an infinite number of possible water distributions even in one dimension. Trial and error, or cost gradient methods based on a few discrete inputs are the only option and can yield only coarse notions about continuous optimal water distributions. 


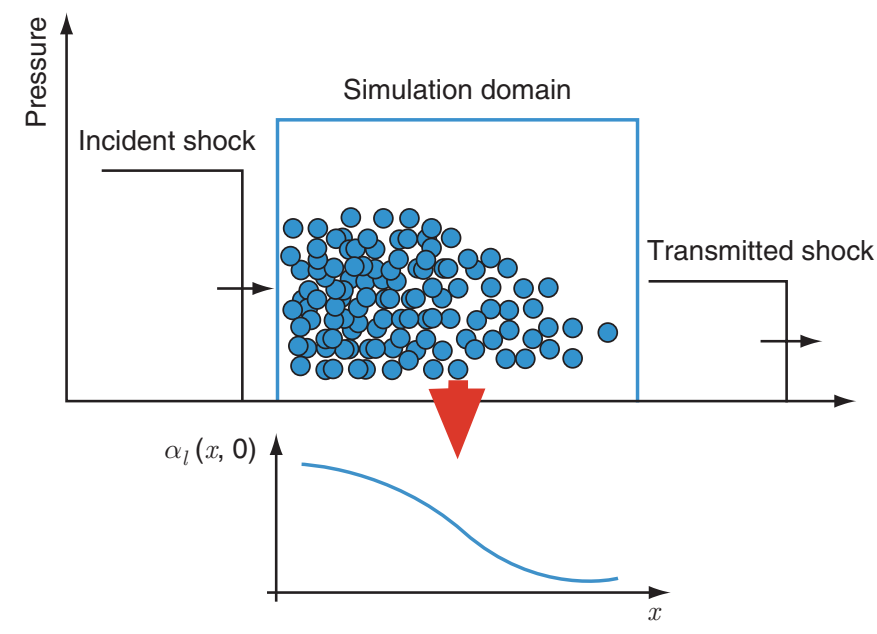

Figure 1. Physical diagram of simulated interaction.

\subsection{New Contributions of Current Work}

The objective of this work is to develop a computational tool that can directly calculate a droplet optimal control for attenuating a range of blast waves to a desired minimal overpressure. As shown in Figure 1, the water droplets' control action will be the result of the initial distribution of the water volume fraction variable $\alpha_{l}(x, 0)$ and the initial droplet diameter. The optimal control $\alpha_{l}^{*}(x, 0)$ will be the distribution of water droplets which yields the greatest decrease to the jump in overpressure of the transmitted shock while not using any more water than is necessary. A simplified, singlephase blast attenuation control problem was first formulated, [23], where the control action is a distributed energy sink behind the shock, a simplification for the way droplet vaporization, the dominant dissipative mechanism, diminishes shock strength. In the present work, a more sophisticated two-phase model, given by [4], is presented and an analogous control problem is formulated in which the control takes the form of the free initial distribution of water droplets which evolves dynamically according to the two-phase model. These control problems are new formulations and the algorithmic solutions developed in order to satisfy all necessary conditions have aspects new to computational results for unsteady shock wave attenuation. First, the mathematical framework of both the two-phase flow model and the optimal conditions will be presented, followed by numerical methods for solving the flow and the control algorithm. Examples of engineering results are given which can be obtained for a given incident blast wave boundary condition in one dimension.

\section{TWO-PHASE FLOW MODEL}

The vast majority of the two-phase flow model is based on the work of Saurel and Abgrall [4, 24]. It consists of six balance laws and a seventh non-conservative PDE for the volume fraction variable. The model was chosen because it maintains a hyperbolic structure and is applicable to physical phenomena of interest, water droplet-shock interactions. This is achieved by considering both phases or fluids as compressible, by assuming many droplets per cell and therefore homogenized interface conditions and by solving the same set of equations everywhere in space.

The state vector of the flow is again denoted as $U$. The subscripts ${ }_{g}$ and ${ }_{l}$ denote gas and liquid respectively. Equations (2) and (3) give the two-phase dynamical system.

$$
\begin{gathered}
U=\left(\alpha_{g}, \alpha_{g} \rho_{g}, \alpha_{g} \rho_{g} u_{g}, \alpha_{g} \rho_{g} E_{g}, \alpha_{l} \rho_{l}, \alpha_{l} \rho_{l} u_{l}, \alpha_{l} \rho_{l} E_{l}\right)^{T} \\
\frac{\partial \alpha_{g}}{\partial t}+V_{i} \frac{\partial \alpha_{g}}{\partial x}=0
\end{gathered}
$$




$$
\frac{\partial}{\partial t}\left(\begin{array}{c}
\alpha_{g} \rho_{g} \\
\alpha_{g} \rho_{g} u_{g} \\
\alpha_{g} \rho_{g} E_{g} \\
\alpha_{l} \rho_{l} \\
\alpha_{l} \rho_{l} u_{l} \\
\alpha_{l} \rho_{l} E_{l}
\end{array}\right)+\frac{\partial}{\partial x}\left(\begin{array}{c}
\alpha_{g} \rho_{g} u_{g} \\
\alpha_{g} \rho_{g} u_{g}^{2}+\alpha_{g} P_{g} \\
u_{g}\left(\alpha_{g} \rho_{g} E_{g}+\alpha_{g} P_{g}\right) \\
\alpha_{l} \rho_{l} u_{l} \\
\alpha_{l} \rho_{l} u_{l}^{2}+\alpha_{l} P_{l} \\
u_{l}\left(\alpha_{l} \rho_{l} E_{l}+\alpha_{l} P_{l}\right)
\end{array}\right)=\left(\begin{array}{c}
0 \\
P_{i} \\
P_{i} V_{i} \\
0 \\
-P_{i} \\
-P_{i} V_{i}
\end{array}\right) \frac{\partial \alpha_{g}}{\partial x}+S(U)
$$

The gas phase obeys the ideal gas equation of state while the stiffened gas equation of state is used for the liquid.

$$
\begin{gathered}
\rho_{g} e_{g}=\frac{P_{g}}{\gamma-1} \\
\rho_{l} e_{l}=\frac{P_{l}+\pi_{l} \gamma_{l}}{\gamma_{l}-1}
\end{gathered}
$$

$\gamma=1.4$ is the gas constant of air, $\gamma_{l}=4.4$ is the analogous constant for water and $\pi_{l}=6 \cdot 10^{8} \mathrm{~Pa}$ is the stiffening constant that makes large changes in liquid pressure produce almost no changes in density. Equations (6) and (7) are closure relations for the internal energy $\rho e$ to the total energy $\rho E$, per unit volume, for each phase.

$$
\begin{gathered}
\rho_{g} E_{g}=\rho_{g} e_{g}+\frac{1}{2} \rho_{g} u_{g}^{2} \\
\rho_{l} E_{l}=\rho_{l} e_{l}+\frac{1}{2} \rho_{l} u_{l}^{2}
\end{gathered}
$$

The volume fraction will propagate at a mean inter-facial velocity which is a center-of-mass estimate given in eqn (8).

$$
V_{i}=\frac{\alpha_{g} \rho_{g} u_{g}+\alpha_{l} \rho_{l} u_{l}}{\alpha_{g} \rho_{g}+\alpha_{l} \rho_{l}}
$$

$E_{i}$ and $P_{i}$ are volume averages of total energy and pressure respectively at the interface shown in eqn (9).

$$
\begin{aligned}
& E_{i}=\alpha_{g} E_{g}+\alpha_{l} E_{l} \\
& P_{i}=\alpha_{g} P_{g}+\alpha_{l} P_{l}
\end{aligned}
$$

The sum of the volume fractions of each phase will always equal 1 everywhere in the onedimensional simulation domain. When either volume fraction tends toward zero, the 1D Euler system is recovered.

$$
\alpha_{g}+\alpha_{l}=1
$$

Let $\tilde{U}$ be the state vector in primitive form in eqn (11). The two-phase system can then be written in non-conservative form in eqns (12-13).

$$
\tilde{U}=\left(\alpha_{g}, \rho_{g}, u_{g}, P_{g}, \rho_{l}, u_{l}, P_{l}\right)^{T}
$$




$$
\begin{gathered}
\frac{\partial \tilde{U}_{i}}{\partial t}+\tilde{A}_{i j}(\tilde{U}) \frac{\partial \tilde{U}_{j}}{\partial x}=S_{i}(\tilde{U}) \\
\tilde{A}(\tilde{U})=\left(\begin{array}{ccccccc}
V_{i} & 0 & 0 & 0 & 0 & 0 & 0 \\
\frac{\rho_{g}}{\alpha_{g}}\left(V_{i}-u_{g}\right) & u_{g} & \rho_{g} & 0 & 0 & 0 & 0 \\
\frac{P_{g}-P_{i}}{\alpha_{g} \rho_{g}} & 0 & u_{g} & 1 / \rho_{g} & 0 & 0 & 0 \\
\frac{\rho_{g} c_{g i}^{2}}{\alpha_{g}}\left(V_{i}-u_{g}\right) & 0 & \rho_{g} c_{g}^{2} & u_{g} & 0 & 0 & 0 \\
\frac{\rho_{l}}{\alpha_{l}}\left(V_{i}-u_{l}\right) & 0 & 0 & 0 & u_{l} & \rho_{l} & 0 \\
\frac{P_{l}-P_{i}}{\alpha_{l} \rho_{l}} & 0 & 0 & 0 & 0 & u_{l} & 1 / \rho_{l} \\
\frac{\rho_{l} c_{l i}^{2}}{\alpha_{l}}\left(V_{i}-u_{l}\right) & 0 & 0 & 0 & 0 & \rho_{l} c_{l}^{2} & u_{l}
\end{array}\right)
\end{gathered}
$$

The source term $H(U)$ which multiplies $\frac{\partial \alpha_{g}}{\partial x}$ in eqn (3) has been coupled into the Jacobian matrix in eqn (13) and the two-phase quasi-linear system now has simply the form shown in eqn (12).

With this fortunate manipulation, the seven eigenvalues can be found by diagonalizing $\tilde{A}(\tilde{U})$. The characteristic speeds are $\left(V_{i}, u_{g}, u_{g}-c_{g}, u_{g}+c_{g}, u_{l}, u_{l}-c_{l}, u_{l}+c_{l}\right)$, where $c_{g}$ and $c_{l}$ are the local speeds of sound in the gas and liquid phases respectively. The velocity of the phase interface is the seventh characteristic wave speed of the system. All are distinct except locally where they are degenerately zero.

The source vector $S(U)$ is broken up into three separate interactions as defined in eqn (14).

$$
S(U)=M H(U)+V R(U)+P R(U)=\left(\begin{array}{c}
0 \\
\dot{m} \\
\dot{m} V_{i} \\
\dot{m}\left(L_{h v}+E_{i}\right)+Q_{i} \\
-\dot{m} \\
-\dot{m} V_{i} \\
-\dot{m}\left(L_{h v}+E_{i}\right)-Q_{i}
\end{array}\right)+\left(\begin{array}{c}
0 \\
0 \\
F_{d} \\
F_{d} V_{i} \\
0 \\
-F_{d} \\
-F_{d} V_{i}
\end{array}\right)+\left(\begin{array}{c}
\mu\left(P_{g}-P_{l}\right) \\
0 \\
0 \\
-\mu P_{i}\left(P_{g}-P_{l}\right) \\
0 \\
0 \\
\mu P_{i}\left(P_{g}-P_{l}\right)
\end{array}\right)
$$

The source vector for mass and heat exchange between the phases is denoted as $M H(U)$. The source terms for velocity and pressure equilibration are $V R(U)$ and $P R(U)$ respectively. As is pointed out in [4], an interface separating two phases must reach the same pressure through microscopic interactions. Indeed, without enforcing this condition, notions of thermodynamic properties such as temperature cannot be determined and numerical oscillations due to pressure differences will grow without significant artificial dissipation [25].

The equilibrium condition $P_{g}=P_{l}$ is chosen thereby neglecting the effect of surface tension. The microscopic pressure equilibration causes a volume and internal energy variation of each phase. Isolating the $\mathrm{ODE} \frac{\partial U}{\partial t}=P R(U)$ in eqn (14) yields conditions better suited to computation.

$$
\begin{gathered}
\frac{\partial \alpha_{g}}{\partial t}=\mu\left(P_{g}-P_{l}\right) \\
\frac{\partial \alpha_{g} \rho_{g} E_{g}}{\partial t}=-\mu P_{i}\left(P_{g}-P_{l}\right)
\end{gathered}
$$




$$
\frac{\partial \alpha_{g} \rho_{g} E_{g}}{\partial t}=-\mu P_{i}\left(P_{g}-P_{l}\right)
$$

Inserting eqn (15) into eqns (16-17) reduces the ODE system to eqns (18-19).

$$
\begin{aligned}
& \frac{\partial \alpha_{g} \rho_{g} E_{g}}{\partial t}=P_{i} \frac{\partial \alpha_{g}}{\partial t} \\
& \frac{\partial \alpha_{g} \rho_{l} E_{l}}{\partial t}=-P_{i} \frac{\partial \alpha}{\partial t}
\end{aligned}
$$

In a closed system, this is just the first law of thermodynamics for an isentropic transformation. Time integration of both sides yields conditions given in eqn (20) and (21) whose solution is achieved via a Newton method which iteratively solves for $\alpha_{g}^{P R}$.

$$
\begin{gathered}
\left(\alpha_{g} \rho_{g} E_{g}\right)^{P R}-\left(\alpha_{g} \rho_{g} E_{g}\right)^{0}=\int_{\alpha_{g}^{0}}^{\alpha_{g}^{P R}} P_{i} \cdot d \alpha_{g} \\
\left(\alpha_{l} \rho_{l} E_{l}\right)^{P R}-\left(\alpha_{l} \rho_{l} E_{l}\right)^{0}=-\int_{\alpha_{g}^{0}}^{\alpha_{g}^{P R}} P_{i} \cdot d \alpha_{g}
\end{gathered}
$$

The superscript ${ }^{P R}$ denotes the solution to the ODE for pressure relaxation while ${ }^{0}$ denotes the starting value which comes from the solution to the balance PDE at the current time step. The drag force $F_{d}$ exerted by the gas onto a spherical water droplet, which is responsible for the $V R(U)$ source term, is given by the empirical drag law in eqn (22) where $D_{p}$ is the diameter of the water droplet.

$$
F_{d}=C_{d} \rho_{g} D_{p}^{2} \alpha_{l}\left(u_{g}-u_{l}\right)^{2}
$$

The droplets' diameter is a dynamic variable distributed in space with the assumption of locally mono-dispersed droplets. $C_{d}$ is a constant drag coefficient.

The exchange of mass and heat between the phases will be mainly due to vaporization of liquid water droplets by the surrounding gas. The rate of gaseous mass production in the form of water vapor from the liquid phase, $\dot{m}$, is defined in eqn (23).

$$
\dot{m}=\rho_{H_{2} \mathrm{Ov}}\left(P_{g}\right) \dot{\alpha}_{l}
$$

The rate of change of the volume fraction of water within a fixed volume of space $\Delta x^{3}$ is related to the diameter rate of change via eqn (24).

$$
\dot{\alpha}_{l}=\frac{S_{p}}{\Delta x^{3}} \frac{\partial D_{p}}{\partial t}
$$

$S_{p}$ denotes the total surface area of droplets per a fixed volume defined in eqn (25).

$$
S_{p}=4 \pi\left(\frac{D_{p}}{2}\right)^{2} N_{p}
$$


$N_{p}$ is the number of droplets per the same fixed volume as is uniquely determined by the volume fraction of water $\alpha_{l}$ for mono-dispersed droplets.

$$
N_{p}=\alpha_{l} \cdot \frac{\Delta x^{3}}{\frac{4}{3} \pi\left(\frac{D_{p}}{2}\right)^{3}}
$$

The evolution of droplet size needed in eqn (26) is determined by the rate of vaporization. In this work, the Empirical-Beta vaporization law is implemented with constant values coming from [12].

$$
\begin{gathered}
\frac{\partial D_{p}^{2}}{\partial t}=-\beta\left(T_{g}\right) \\
\beta\left(T_{g}\right)=7600\left(1+7.4 \cdot 10^{-7}\left(T_{g}-300 K\right)_{+}\right)^{2.7548} \mu m^{2} / s
\end{gathered}
$$

As will be demonstrated in the Results section, the range of pressure magnitude for the flows of interest to the IOP attenuation problem are from 1-10 atm. According to steam tables [26], the water vapor density will vary by an order of magnitude over this pressure range. Consequently, water droplet vaporization will be much more effective to high pressure shocks. Equation (29) is the least-squares quadratic fit to the steam table data for water vapor density as a function of the surrounding gas pressure.

$$
\rho_{\mathrm{H}_{2} \mathrm{Ov}}\left(P_{g} / \mathrm{atm}\right) \approx-.0024344 \cdot P_{g}^{2}+.5368 \cdot P_{g}-.077246 \mathrm{~kg} / \mathrm{m}^{3}
$$

The last term in the $M H(U)$ vector, $Q_{i}$, is the rate of heat exchange between phases at the interface, given in eqn (30) where $h$ is the heat transfer coefficient.

$$
Q_{i}=h S_{p}\left(T_{g}-T_{l}\right)
$$

For water droplets of diameter $D_{p}, h=\frac{N u \lambda}{D_{p}}$ where $N u$ is the Nusselts Number and $\lambda=0.6 \mathrm{~W} / \mathrm{mK}$, is the thermal conductivity of water.

\subsection{Well-Posedness}

The eigenvalues of $\tilde{A}$ above are $\left(V_{i}, u_{g}, u_{g}-c_{g}, u_{g}+c_{g}, u_{l}, u_{l}-c_{l}, u_{l}+c_{l}\right)$. From the definition for interface velocity and sound speeds it can be seen that all these eigenvalues are real and distinct if a few conditions hold. If there are regions in our domain where a single phase is completely absent, then the interface velocity will be degenerate to the velocity of the phase $100 \%$ present. Therefore, our calculation restricts the volume fraction of either phase to remain above a certain small threshold. As with the singlephase Euler system in one dimension, well-posedness can be shown by the existence of a real, positivedefinite symmetrizer. It was shown by [27] that a necessary condition on the positive-definiteness of the symmetrizer depends on the positiveness of a certain constitutive variable that should always physically be positive such as density, temperature etc. The two-phase system has the same requirement. The Jacobian matrix in eqn (13) is symmetrizeable if, in addition to the restriction that density and temperature remain positive, the volume fraction of each phase remain non-zero. Since our model dictates that the same equations be solved in each cell, no further care is needed to maintain well-posedness.

\section{METHOD OF SOLUTION FOR TWO-PHASE GAS-LIQUID COUPLING}

The two-phase balance equations, point-wise relations, interface quantities and source terms defined in the previous section are the basis for the overarching numeric method used to couple the two-phase system. While defining the mathematical model is essential to describing the optimal control system, the details of the numeric method for solving the flow are summarized for brevity and the reader is referred to [4] for greater detail. 
The sequence of numeric operations which progresses the solution from the $n^{\text {th }}$ to the $(n+1)^{\text {th }}$ time-step are given in eqn (31).

$$
U^{n+1}=L_{s}^{\Delta t / 2} L_{h}^{\Delta t / 2} L_{s}^{\Delta t / 2} U^{n}
$$

The operator $L_{h}$ represents solving the discretization of the hyperbolic system shown in eqn (32).

$$
L_{h}: \frac{\partial U}{\partial t}+F(U)_{x}=H(U) \frac{\partial \alpha_{g}}{\partial x}
$$

The operator $L_{s}$ represents solving the discretization of the three separate source terms system shown in eqns (33-34).

$$
\begin{gathered}
L_{s}: \frac{\partial U}{\partial t}=S(U) \\
L_{s} \equiv L_{M H} \cdot L_{V R} \cdot L_{P R}
\end{gathered}
$$

A conservative Godunov method [28], second-order accurate in space, is used to solve the system in eqn (32). Details on that method are described in [29]. In order to maintain second order accuracy, the solution involving the source term operators must therefore be performed for a half time-step as is shown in eqn (31), [30].

Details on the method of evolution of the droplets' sizes and other properties related to vaporization are provided since they were developed specifically for the current work. The present calculation assumes that droplets are mono-dispersed and spherical. Their initial size is denoted $D_{p}^{0}\left(x_{i}, 0\right)$. When surrounded by hot gas, the droplets will start to vaporize and their diameter will evolve according to eqn (35), a discrete integration of eqn (27).

$$
D_{p}^{2}\left(x_{i}, t^{n+1}\right)=D_{p}^{2}\left(x_{i}, t^{n}\right)-\Delta t \cdot \beta\left(T_{g}\left(x_{i}, t^{n}\right)\right)
$$

Once the new size of the droplets are known, the added amount of gas volume fraction is known.

$$
\alpha_{g}^{n+1}=\alpha_{g}^{n}+\frac{4}{3} \pi\left(\frac{D_{p}^{n+1^{3}}}{2}-\frac{D_{p}^{n^{3}}}{2}\right) N_{p}^{n}
$$

The critical quantity for sinking energy from the gas via vaporization is $\dot{m}$ which is given in discrete form below based on eqn (37).

$$
\dot{m}\left(x_{i}, t^{n+1}\right)=\rho_{H 2 O v}\left(P_{g}\left(x_{i}, t^{n+1}\right) / a t m\right)\left(\alpha_{g}\left(x_{i}, t^{n+1}\right)-\alpha_{g}\left(x_{i}, t^{n}\right)\right)
$$

The density of water is calculated from eqn (29).

With the additional mass of water vapor in the gas phase, the gas density will increase according to eqn (38) while the density of liquid water does not change.

$$
\rho_{g}^{n+1}=\left(\alpha_{g}^{n} \rho_{g}^{n}+\dot{m}\right) / \alpha_{g}^{n+1}
$$

The last source term operator in eqn (34), $L_{M H}$, can now be integrated since the terms defining $M H(U)$ in eqn (14) are now known at the current time-step.

\section{DERIVATION OF NECESSARY CONDITIONS OF OPTIMAL SYSTEM}

Optimal control of fluid dynamics has undergone rapid developments during the past two decades [31-33]. Optimal control theory of hyperbolic systems of conservation laws for applications of gas dynamics with shock waves is addressed in $[34,35]$. This section gives a brief derivation of all first order 
necessary conditions of optimality. Let a nonlinear partial differential operator $N$, which operates on a state vector $U$ and control vector $z$ take the form of eqn (39).

$$
N(U, z)=\frac{\partial U_{i}}{\partial t}+A_{i j}(U) \frac{\partial U_{j}}{\partial x_{k}}-S_{i}(U)-z_{i}=0
$$

After the dynamical system is known, a cost, or objective, functional must be intelligently defined. In the most general framework there can be a cost associated with the initial data $I$, a cost involving the final data $K$ and a running $\operatorname{cost} L$ that accumulates over the time interval of the control problem [0,T]. So far, initial and final data can still be free or fixed, no restriction has been made. The cost functionals describe the objective associated with how these parameters are distributed. Let $J$ be the total cost functional.

$$
J=I(U(., 0))+K(U(., T))+\int_{0}^{T} L(U, z) d t
$$

To incorporate the constraint in the minimization, $N$ is multiplied by a generic continuous vector $V(x, t)$ and added to $J$. The problem then becomes minimizing the augmented cost functional $\tilde{J}$.

$$
\tilde{J}=I(U(., 0))+K(U(., T))+\int_{0}^{T} L(U, z)+(V, N(U, z)) d t
$$

It is standard to refer to $V_{i}(x, t)$ as the costate or adjoint vector. It is a $m$-dimensional vector since there is an adjoint state for every state variable. The notation $(\cdot, \cdot)$ signifies a spatial integral.

Let $\left(z^{*}, U^{*}, V^{*}, T^{*}\right)$ be the optimal control, state vector, adjoint vector and final time respectively. Let a perturbed control function $\delta z$ be added to the optimal control solution so that:

$$
z=z^{*}+\epsilon \delta z
$$

where $\epsilon>0$ is a small constant. The perturbed optimal control introduces a perturbation to the optimal state and adjoint state:

$$
\begin{aligned}
& U=U^{*}+\epsilon \delta U \\
& V=V^{*}+\epsilon \delta V
\end{aligned}
$$

Another way to see $\delta U$ which will be useful shortly is:

$$
\delta U=\left.\frac{d}{d \epsilon} U\left(z^{*}+\epsilon \delta z\right)\right|_{\epsilon=0}
$$

It has been pointed out in the literature [36-38] that if the state variables have shocks, a perturbation is not small in the neighborhood of the shock and does not have the vanishing properties as $\epsilon \rightarrow 0$. A slight increase in the amplitude behind the shock perturbs the shock speed and therefore also the location of the shock front. This causes small perturbations to induce variations on the order of the jump across the shock. The presented method of solution avoids this issue, as will be demonstrated later. Since only decreasing the amplitude of a shock wave is desired it is apparent that any realistic control action will only slow the shock wave down. The target state $Q(x)$ and final time penalty will be constructed in such a way that all variations of the solution will occur upstream of the shock front only. Matching the simulated pressure profile under control action with the target final state near the shock front will occur by allowing the final time to be free. Henceforth, it can be assumed that all variations are taken in smooth regions of the flow and that the solution procedure will not depend on an additional shock location variable and corresponding adjoint state, nor is a different type of variation required.

The first order necessary optimal condition that must be satisfied by a perturbed control, state, adjoint state and final time $(\delta z, \delta U, \delta V, \delta t)$ is: 


$$
\left.\frac{d}{d \epsilon} \tilde{J}\left(z^{*}+\epsilon \delta z\right)\right|_{\epsilon=0}=0
$$

Equations (47-50) are what is obtained after expanding all of the terms and grouping the ones with like variations. $x_{R}$ and $x_{L}$ denote the right and left points of the spatial boundary in a single dimension.

$$
\begin{aligned}
& \int_{0}^{T}\left(\frac{\partial L}{\partial z}-V^{*}, \delta z(x, t)\right)+ \\
& \left(-\frac{\partial V_{j}^{*}}{\partial t}+\left(\frac{\partial A_{i j}}{\partial U_{k}} \frac{\partial U_{k}}{\partial x}-\frac{\partial A_{i j}}{\partial x}\right) V_{i}^{*}-A_{i j} \frac{\partial V_{i}^{*}}{\partial x}-\frac{\partial S_{i}}{\partial U_{j}} V_{i}^{*}+\frac{\partial L}{\partial U_{j}}, \delta U_{j}\right)+ \\
& -\left.\frac{\partial}{\partial x}\left[\left(A_{i j} V_{i}^{*}, \delta U_{j}\right)\right]\right|_{x=x_{L}} ^{x}+\left.\left(L+\left(V^{*}, N\left(U^{*}, z^{*}\right)\right)\right)\right|_{t=T} \delta t=0 \\
& \left(\frac{\partial I}{\partial U}-V^{*}(x, 0), \delta U(x, 0)\right)=0 \\
& \left(\frac{\partial K}{\partial U}+V^{*}(x, T), \delta U(x, T)\right)=0
\end{aligned}
$$

Recall that the final time is free to vary. Therefore, a necessary condition for the final time must be constructed. This is known as the Transversality condition. The variation of the state at the final time will have a first order term expanded in space $\delta U(x, T)=\delta U+\frac{\partial U(x, T)}{\partial t} \delta t$. Therefore, there is a change to the final time boundary condition.

$$
\left(\delta U(x, T)+\frac{\partial U(x, T)}{\partial t} \delta t, \frac{\partial K}{\partial U}+V^{*}\right)=\left(\delta U, \frac{\partial K}{\partial U}+V^{*}\right)+\left(\delta t, \frac{\partial U}{\partial t}\left(\frac{\partial K}{\partial U}+V^{*}\right)\right)
$$

The pseudo-Hamiltonian is defined in general in eqn (51).

$$
H=L+\left(V, \frac{\partial U}{\partial t}\right)
$$

Using this definition, the Transversality Condition takes it's familiar form in eqn (52).

$$
\left(\delta t,\left.H\right|_{t=T^{*}}+\frac{d K}{d t}\right)=0
$$

\section{TWO-PHASE CONTROL PROBLEM FORMULATION AND SOLUTION}

The solution procedure will iteratively reach a control, state, adjoint and final time that satisfy all first order necessary conditions. An adjoint PDE must be solved backward in time to fix an initial condition on a free state variable. The cost functional $J$, given in continuous form in eqn (53) and shown in discrete form in eqn (56), will be minimized while all constraints of the two-phase model are obeyed.

$$
J=\int_{\Omega} \frac{a}{2}\left(\alpha_{l}(x, 0)\right)^{2} d x+\int_{\Omega s} \frac{b}{2}\left(P_{g}(x, T)-Q(x)\right)_{+}^{2} d x
$$


Therefore, the initial penalty $I$ and final time penalty $K$ are defined by eqns (54-55). An example of an uncontrolled blast profile, target state and the final time pressure profile at some iteration in the solution procedure are illustrated in Figure 2.

$$
\begin{gathered}
I(U(., 0))=\int_{\Omega} \frac{a}{2}\left(\alpha_{l}(x, 0)\right)^{2} d x \\
K(U(., T))=\frac{b}{2} \int_{\Omega s}\left(P_{g}(x, T)-Q(x)\right)_{+}^{2} d x \\
J=\sum_{i=1}^{M} \frac{a}{2} \alpha_{l}^{2}\left(x_{i}, t^{0}\right) \Delta x+ \\
\sum_{x_{i} \in \Omega_{s}} \frac{b}{2} \max \left(P_{g}\left(x_{i}, t^{N}\right)-Q\left(x_{i}\right), 0\right)^{2} \Delta x
\end{gathered}
$$

There is no running penalty necessary since the cost associated with control action is penalized at the initial time and the cost associated with missing the target pressure profile need only be assessed at the final time. Recall the state vector in primitive form, $\tilde{U}=\left(\alpha_{g}, \rho_{g}, u_{g}, P_{g}, \rho_{l}, u_{l}, P_{l}\right)^{T}$ and the constraint on the free initial data $\alpha_{g}+\alpha_{l}=1$ and $0<\alpha_{l}<1$, throughout the simulation domain $\Omega$. The rest of the state variables have fixed initial conditions that are given in eqn (57).

$$
\begin{array}{ll}
\rho_{g}(x, 0)=1.27 \mathrm{~kg} / \mathrm{m}^{3} & \rho_{l}(x, 0)=10^{3} \mathrm{~kg} / \mathrm{m}^{3} \\
u_{g}(x, 0)=0 \mathrm{~m} / \mathrm{s} & u_{l}(x, 0)=0 \mathrm{~m} / \mathrm{s} \\
P_{g}(x, 0)=10^{5} \mathrm{~Pa} & P_{l}(x, 0)=10^{5} \mathrm{~Pa}
\end{array}
$$

The method for obtaining the discretized flow field was described in the previous section. To proceed with numerical conditions based on the optimal control system, it is assumed that an entire flow field $U_{k}^{l}\left(x_{i}, t^{n}\right)$ is known for all spatial indices $i$, temporal indices $n$, variable indices $k$ and overall solution iterations $l$. The vast majority of the additional computational complexity required to satisfy all conditions of the optimal system is solving the adjoint PDE.

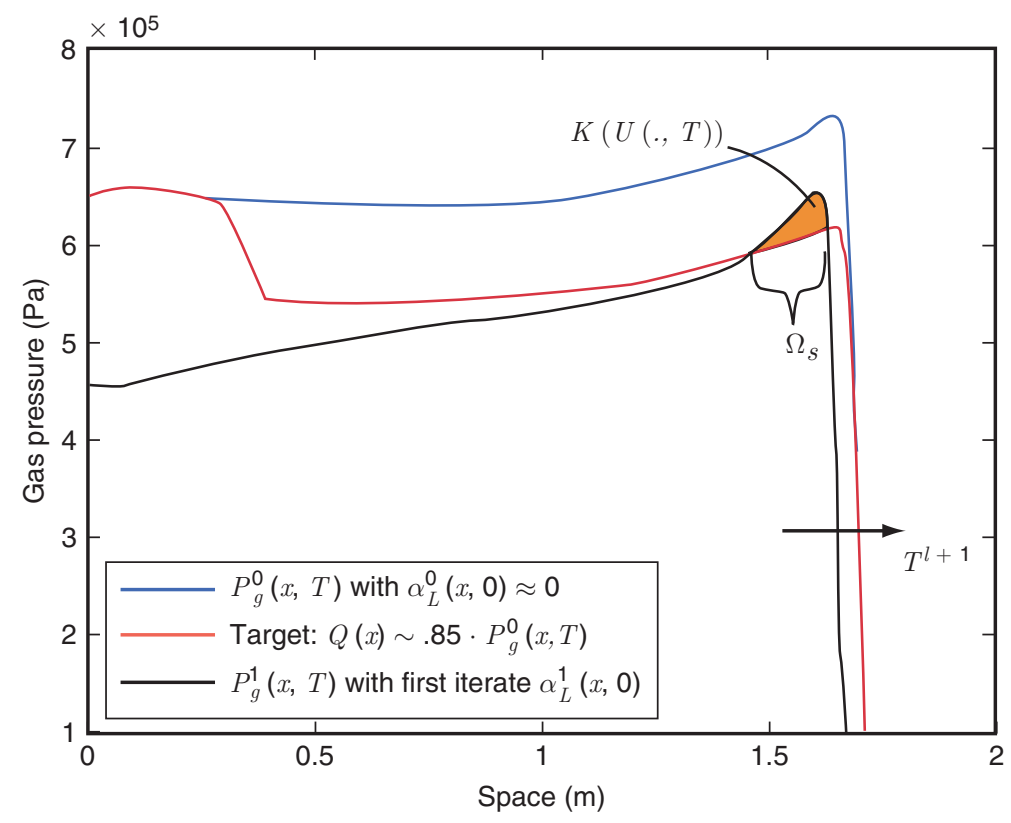

Figure 2. Illustration of Iterative Procedure. 

Application to Ignition Overpressure in Launch Vehicles

It is convenient to define the target state in terms of the pressure. Since the Jacobian has already been provided in the primitive basis in [4] the adjoint PDE is simpler to derive in that same basis. The nontrivial elements of the matrices are given in [39]. The necessary condition on the adjoint vector at the final time is given for all $x_{i} \in \Omega_{s}$. As shown in Figure 2, $\Omega_{s}$ is defined as the region in space where an iterate of the final time pressure is greater than the target state.

$$
V_{k}^{l}\left(x_{i}, T^{l}\right)=\left(\begin{array}{ll}
b \cdot \max \left(\left(P\left(x_{i}, T^{l}\right)-Q\left(x_{i}\right)\right), 0\right) & k=4 \\
0 & k \neq 4
\end{array}\right.
$$

Starting with the final time boundary condition in eqn (58), the adjoint PDE can be solved backward in time to arrive at a value for $V_{1}^{l}\left(x_{i}, 0\right)$. From the necessary condition given in eqn (48), the volume fraction of liquid at each point in space will be iterated, within the constraints, via a fixed-point Newton method shown in eqn (59).

$$
\alpha_{g}^{l+1}\left(x_{i}, t^{0}\right)=\alpha_{g}^{l}\left(x_{i}, t^{0}\right)-\frac{a\left(1-\alpha_{g}^{l}\left(x_{i}, t^{0}\right)\right)-V_{1}^{l}\left(x_{i}, t^{0}\right)}{-a} \alpha_{l}^{l+1}=1-\alpha_{l}^{l+1}
$$

A survey of the literature on adjoint-based methods for optimal control [40-43] gave insight on how to construct a solution algorithm. However, there were no methods specifically for distributed control with free initial and final data and final time for unsteady shock attenuation.

To solve for the optimal final time, the Transversality Condition for the two-phase system, given in eqn (52), is defined as a continuous function $f(T)$ with the final time as the independent variable. Then $f\left(T^{*}\right)=0$ at the optimal final time $T^{*}$. The optimal final time, $T^{*}$, can be solved iteratively with the Newton method from using the discretization of $f$ in eqn (60).

$$
f\left(T^{l}\right)=\sum_{i=1}^{m}\left(P_{g}\left(x_{i}, t^{N}\right)-Q\left(x_{i}\right)\right) \frac{P_{g}\left(x_{i}, t^{N}\right)-P_{g}\left(x_{i}, t^{N-1}\right)}{\Delta t} \Delta x
$$

The definition of $\frac{d f}{d t}$ and its discretization are also given in [39]. The overall algorithm which is used to satisfy all of the above necessary optimal conditions is shown in block-diagram form in Figure 3.

The continuous form of the adjoint PDE system is shown as the pairing with $\delta U_{j}$ in eqn (47). The system is linear in the adjoint variables. The time derivative of the entire adjoint vector for all space at a discrete time $t^{n}$ is shown in column-upon-column form in eqn (61). Let $m$ be the number of spatial grid points and $k$ be the number of adjoint variables. Then the adjoint vector $V$ at a discrete moment in time will be of size $\mathrm{km}$ by 1 and the matrices in eqn (47) will be of size $\mathrm{km}$ by $\mathrm{km}$. A single component of the adjoint vector, eg. $V_{1}(x, t)$, will be size $m$ by 1 at each time step.

$$
\left.\frac{\partial V_{k}^{l}}{\partial t} \leftrightarrow \frac{\partial}{\partial t}\left(\begin{array}{c}
\left(\begin{array}{c}
V_{1}\left(x_{1}, t^{n}\right) \\
\vdots \\
V_{1}\left(x_{m}, t^{n}\right)
\end{array}\right) \\
\left.V_{2}\left(x_{1}, t^{n}\right)\right) \\
\vdots \\
\vdots \\
V_{k}\left(x_{1}, t^{n}\right) \\
\vdots \\
V_{k}\left(x_{m}, t^{n}\right)
\end{array}\right)\right)
$$




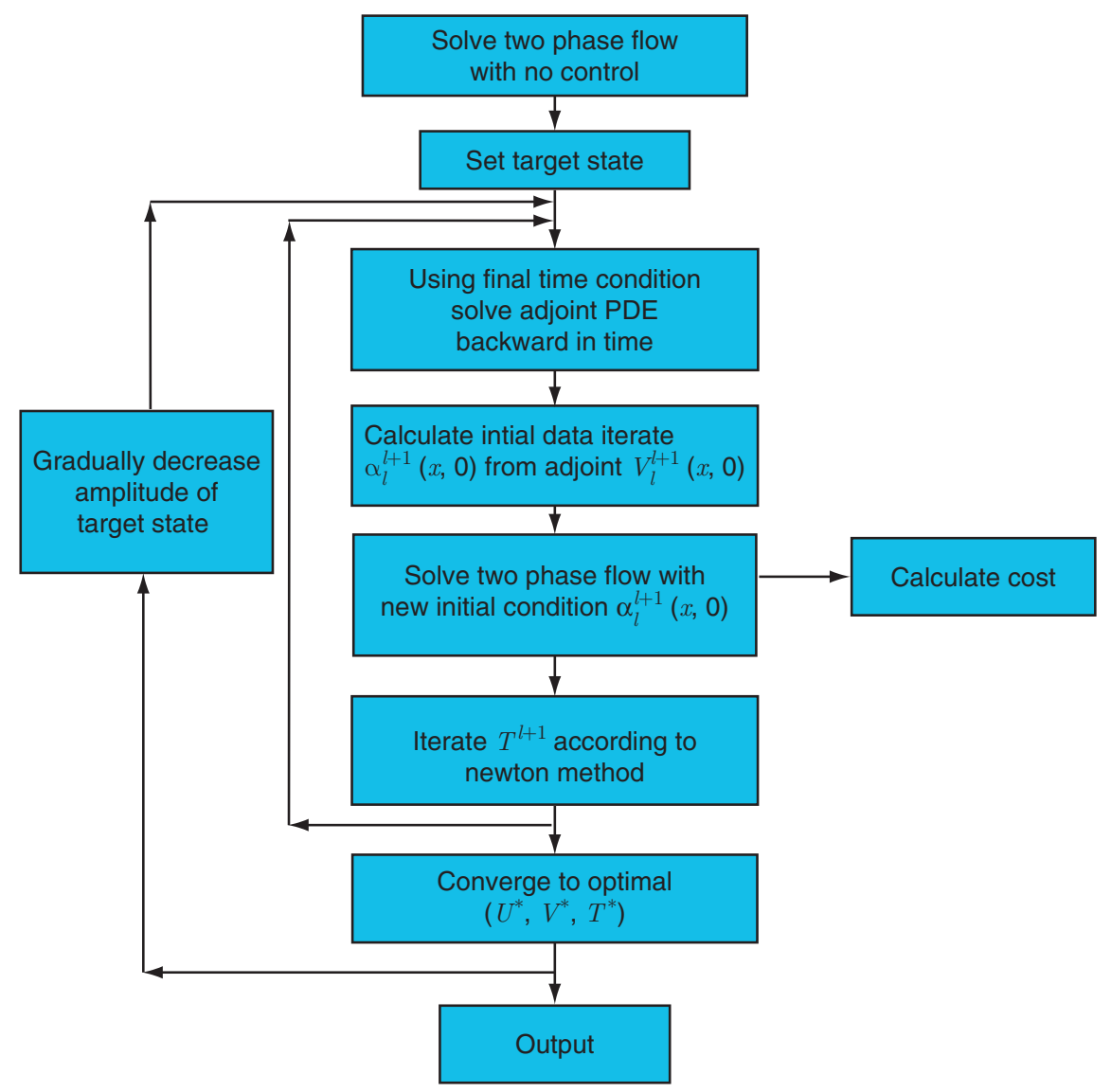

Figure 3. Block diagram of solution procedure for two-phase control calculation.

All of the matrices in eqn (47) have a diagonal-block structure. The Jacobian matrix for all of space at $t^{n}$ is shown in eqn (62).

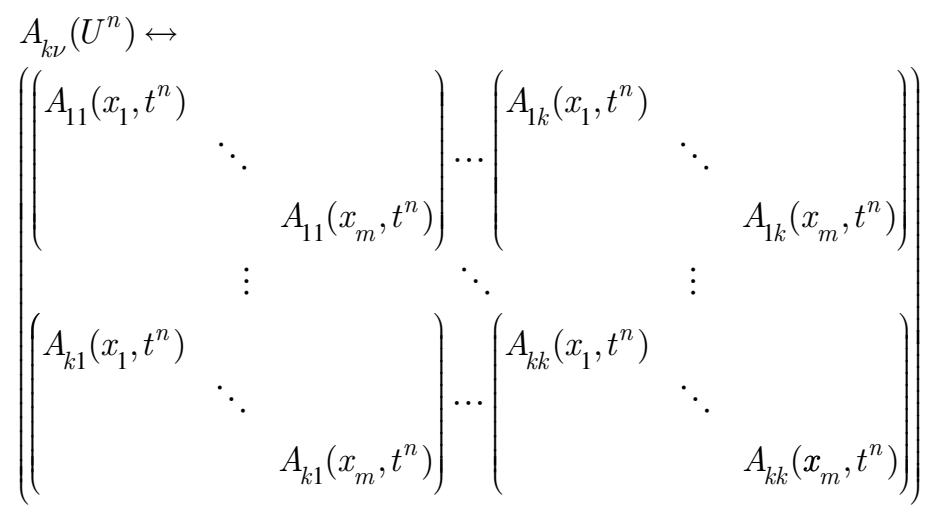

The adjoint PDE is given in discrete form with an explicit integration in eqns (63-64).

$$
\begin{gathered}
\frac{1}{\Delta t} I\left(V_{k}^{n-1}-V_{k}^{n}\right)+R\left(U^{n}\right) V_{i}^{n}=0 \\
R(U)=A \cdot D U+\frac{\partial A}{\partial x}-\frac{\partial A}{\partial U} \cdot \frac{\partial U}{\partial x}+\frac{\partial S}{\partial U}
\end{gathered}
$$


The matrix $D$ is made up of discrete spatial derivative block matrices, central differencing in the domain interior, upwind differencing at the outlet and downwind at the inlet. A single block is shown in eqn (65).

$$
D_{m x m}=\frac{1}{2 \Delta x}\left(\begin{array}{ccccc}
-2 & 2 & & & \\
-1 & 0 & 1 & & \\
& \ddots & \ddots & \ddots & \\
& & -1 & 0 & 1 \\
& & & 2 & -2
\end{array}\right)
$$

For adjoint calculations of a scalar PDE with a discontinuity, it has been shown [44, 45] that a relaxed system with second order dissipation will recover the non-linear PDE in the limit of vanishing viscosity. A small numerical viscosity can stabilize the adjoint solution. These ideas have been extended to fluid dynamics systems in [45] and are implemented in the current work, in a manner which maintains consistency for the two-phase numerical adjoint solutions.

\section{RESULTS}

Data on the shuttle grain and chamber pressure from [46] was input to Cequel [47] a code for steady state rocket property calculations. The output gives the temperature and gas velocity at the nozzle exit plane for a given pressure ratio. Initially, ambient conditions inside the domain are present. The ignition sequence was simulated in 2D using the ESI-Fastran commercial software, [48]. The spatial discretization scheme used was Van Leer's flux vector splitting and was extended to second order accuracy by a Barth limiter. The Barth limiter enforces monotonicity and, therefore, is appropriate for solutions with strong discontinuities. Time integration was fully implicit with a tolerance of $10^{-4}$ in the residuals over a maximum of 20 sub-iterations.

Constant mass-flow boundary conditions equivalent to the steady state exit plane of the rocket nozzle on the shuttle were used in the bottom center of the domain on the right face of the step as shown in Figure 4.a. Mach number is depicted in three snapshots in Figure 4.a, 4.c and 4.e and pressure in Figure 4.b, 4.d, and 4.f. The last frame is roughly $10 \mathrm{~ms}$ after ignition. The bottom left edge of the domain represents the rocket body while the bottom right edge is the centerline of the normal to the nozzle exit plane and a symmetry boundary. All other edges are non-reflecting boundaries.

Flow conditions over time were recorded at two locations marked in Figure 4.a. Point 1 is near the rocket body 2.5 meters above the nozzle and Point 2 is 1.5 meters along the symmetry boundary and the plane normal to the nozzle exit. The conditions at the recorded locations are used as the boundary conditions in both the single- and two-phase control calculations.

Figure 5.a shows the flow conditions over time at Monitor Points 1 near the rocket body and Figure 5.b shows the flow conditions over time for Monitor Point 2 directly downstream of the nozzle.

It is worth mentioning that the two-dimensional flow cannot be truly replicated by an inlet boundary condition to a single-dimensional calculation. Neglecting motion in the transverse direction while taking $(\rho, u, P)$ explicitly means that the resulting driver gas has a lower temperature. This is not discouraging since intuition would suggest that hotter gas has more potential to vaporize water droplets and hence, by the nature of the control action, there is more potential for effectiveness. The goal is to be able to handle a range of blast waves that will plausibly be seen in an IOP environment.

The results for the two-phase control problem were obtained with the algorithm shown in Figure 3. In each case, the spatial grid size was $1 \mathrm{~cm}$ and the time-step was $1 \mu \mathrm{s}$. Figure $6 . \mathrm{a}$ shows the optimal initial condition for the free water volume fraction variable. The inlet condition is given by the data from Monitor Point 1 pictured in Figure 4.a and graphed over time in Figure 5.a. The curves give the optimal water volume fraction distributions for four target states with successively decreasing jumps in pressure at the shock front. The legend tells what percentage of the absolute overpressure of the uncontrolled blast wave was used to define the target state.

Figures 7.a and 7.b are analogous to Figure 6.a and 6.b except that now the driving inlet boundary condition is from Monitor Point 2; which is pictured in Figure 4.a and graphed over time in Figure 5.b. Monitor Point 1 data gives a shock with a little less than a 2:1 jump in pressure at the shock front, and a driving gas temperature of less than $500 \mathrm{~K}$. Monitor Point 2 data gives a shock with about an 8:1 jump in pressure at the shock and a driving gas temperature of more than $1000 \mathrm{~K}$. Several differences in the 
(a)

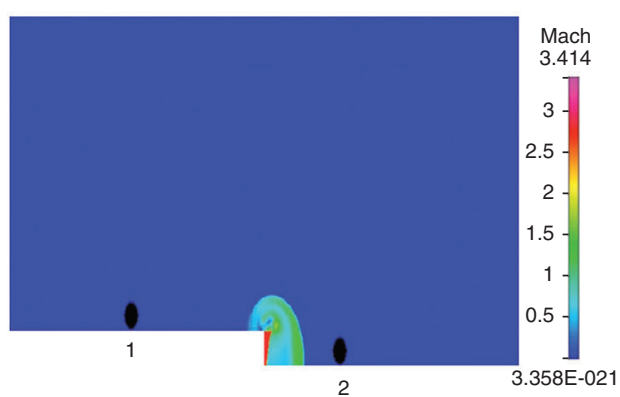

(c)

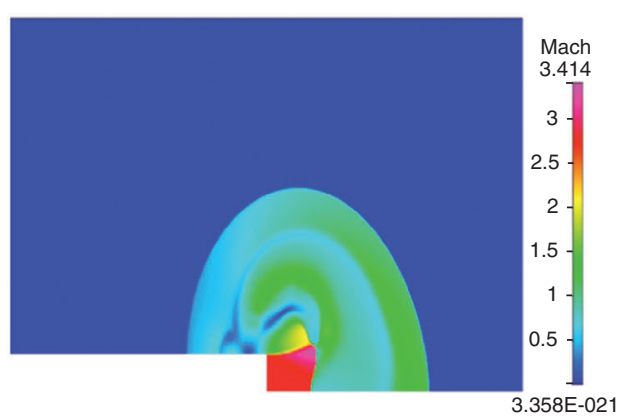

(e)

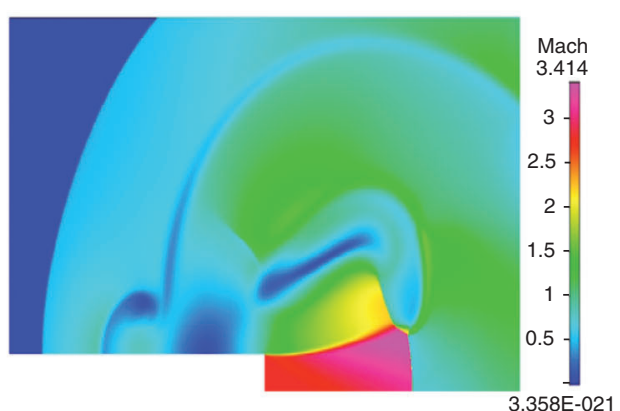

(b)

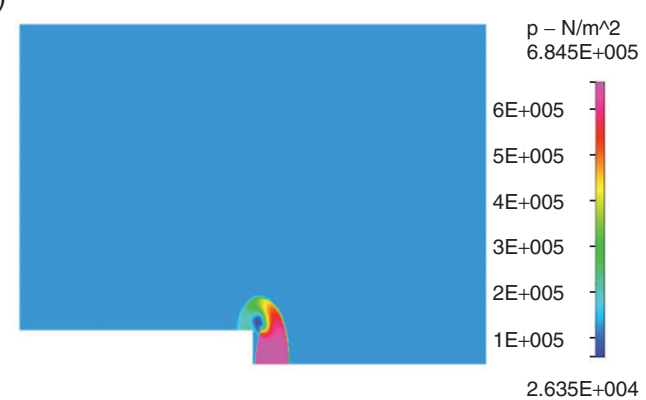

(d)

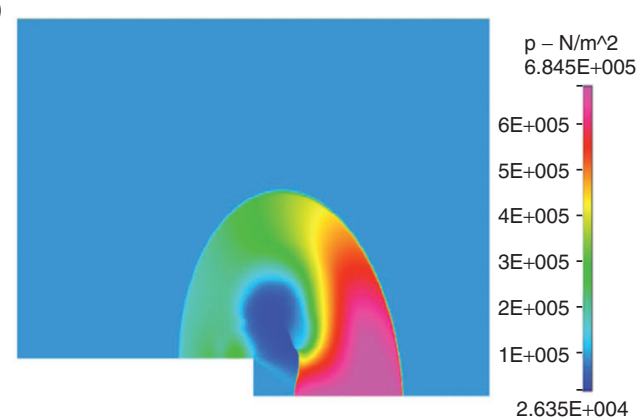

(f)

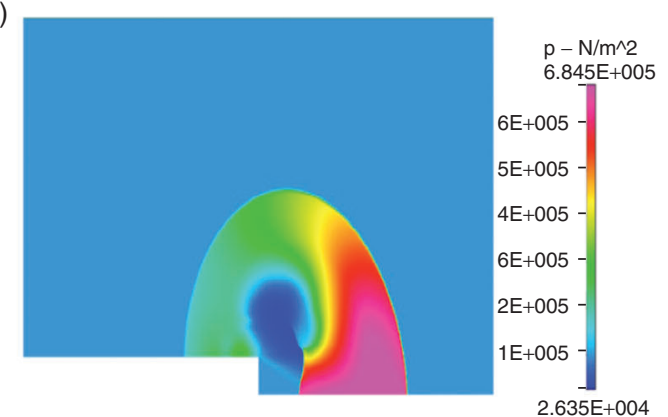

Figure 4. Simulated Shuttle IOP: Mach Number at (1.2 ms, $4 \mathrm{~ms}, 10 \mathrm{~ms})$ after ignition (a, c, e); Pressure at (1.2 ms, $4 \mathrm{~ms}, 10 \mathrm{~ms})$ after ignition (b, d, f).

(a)
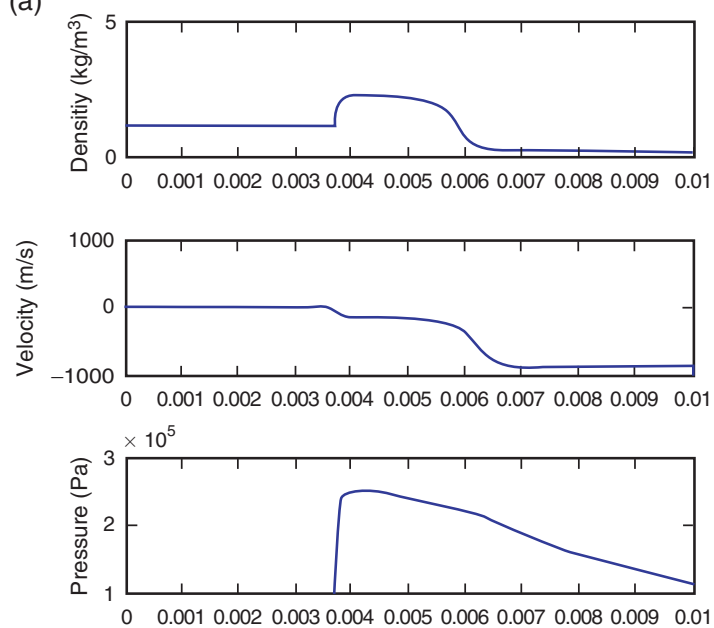

Time (s) (b)
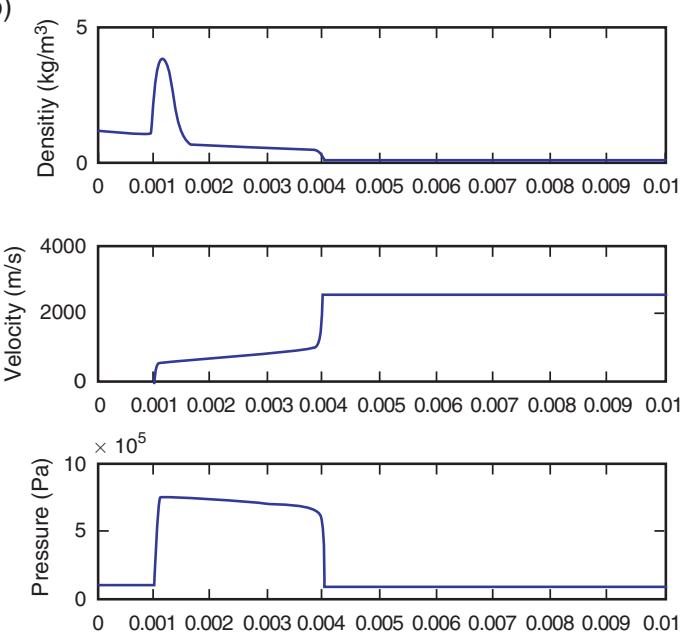

Time (s)

Figure 5. Flow conditions over time for Monitor Point 1 (a) and Monitor Point 2 (b). 
(a)

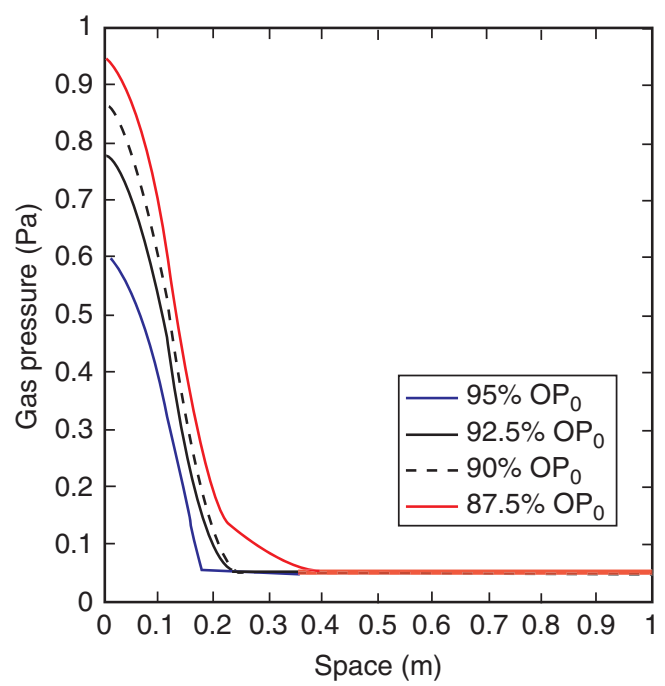

(b)

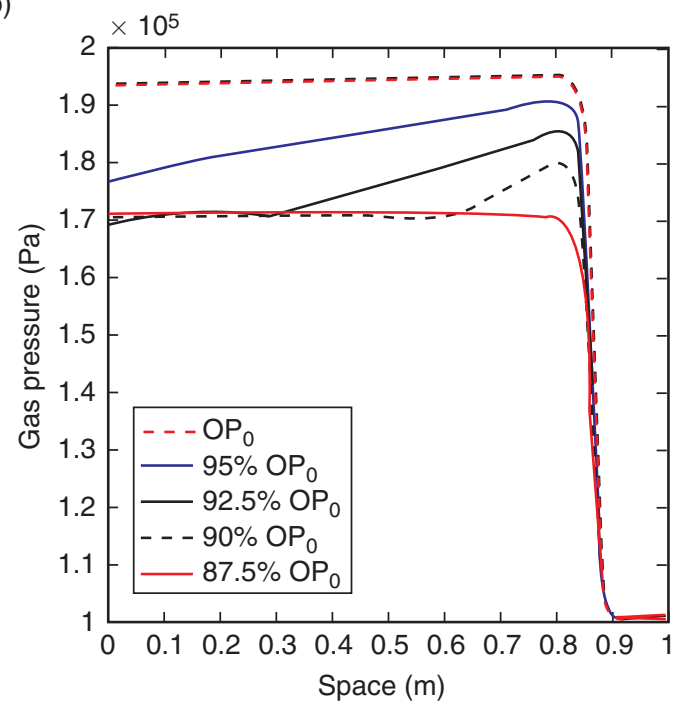

Figure 6. (a) Unconstrained optimal water volume fraction distributions at initial moment in time $\alpha_{l}^{*}(x, 0)$ for MP1 inlet condition (b) Optimal final time pressure profiles $P^{*}\left(x, T^{*}\right)$ resulting from initial data in Figure a.

(a)

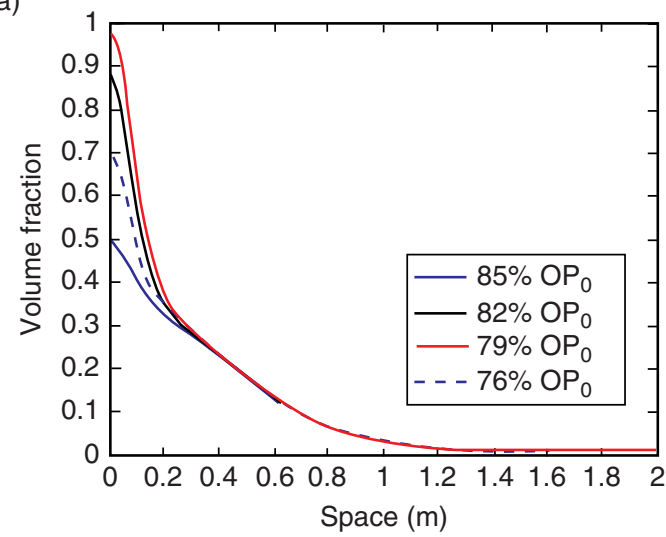

(b)

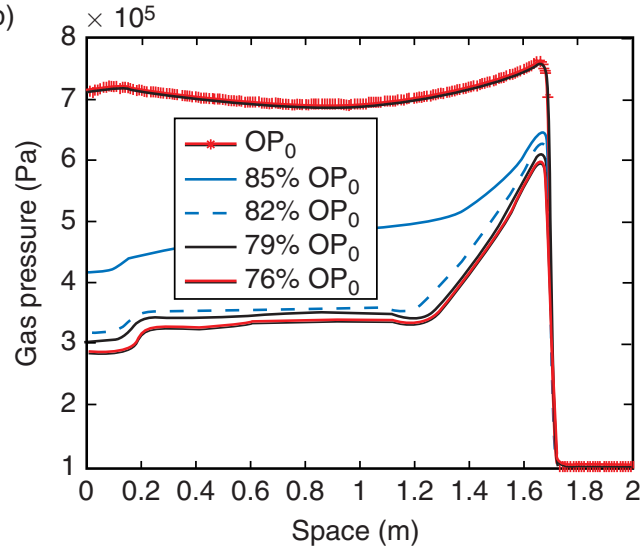

Figure 7. (a) Unconstrained optimal water volume fraction distributions at initial moment in time $\alpha_{l}^{*}(x, 0)$ for MP2 inlet condition (b) Optimal final time pressure profiles $P^{*}\left(x, T^{*}\right)$ resulting from initial data in Figure a.

solution are worth noting as they give insight into how differently the water droplets affect blast waves over the range of overpressure that may be encountered in an IOP environment.

The largest fractional decrease in overpressure achievable in $2 \mathrm{~ms}$ with with the MP1 inlet condition is $87.5 \%$ of the uncontrolled wave, $\left(O P_{0}\right)$ while the largest fractional decrease in overpressure achievable (in the same time interval) with with the inlet condition of MP2 is $76 \%$ of the uncontrolled wave. Notice that the MP2 shock travels nearly twice as far as the MP1 shock. The shape and maximum height of the optimal water distributions for these two cases are nearly the same. This shows that the water droplets are able to affect a greater decrease in shock strength when the shock is driven by hotter gas since hotter gas will vaporize water more quickly and cause a larger decrease in the pressure of the gas pushing the shock. This is illustrated in Figure 6.b and 7.b. Notice that in Figure 6.b, the most attenuated shock profile is flat while this is not true of the most attenuated profile in Figure 7.b. The difference comes from the gas temperature. The lower temperature gas from the MP1 data has entirely cooled to about $300 \mathrm{~K}$ at the end of the simulation which leaving no more potential for droplet vaporization. In contrast, the hotter temperature gas from the MP2 data has not yet entirely cooled to $300 \mathrm{~K}$, meaning that further droplet vaporization is possible and that the pressure profiles have yet to flatten out. 
The convergence of the algorithm is shown in Figure 8.a. Every 25 iterations, the target state's amplitude is ramped down so that optimal solutions that yield ever increasing attenuation levels can be determined in a single execution of code. The history of the final time variable over the iterative solution procedure is shown in Figure 8.b. As the final time changed, the time-step size changed slightly from $1 \mu s$ so that the nearest integer number of time-steps yielded the final time iterate.

If the target amplitude is set too low, first-order variation conditions fail to maintain convergence of the overall algorithm. The argument that the solution is indeed a minimum can be readily illustrated in Figure 8.a. The final time penalty is weighted to a much larger magnitude than the initial penalty thereby ensuring that the solution definitely is one which attenuates the shock wave. At the same time the control action is ever-increasing at a decreasing rate. Therefore, if less control is used, $J$ will increase because $K$ will increase. Similarly if more control is used $J$ will still increase because $I$ will increase and $K$ cannot decrease any further.

Another case was run with an upper bound on the volume fraction of water at $70 \%$. The calculated optimal water distributions are shown in Figure 9.a with the resulting pressure profiles shown in Figure 9.b.

(a)

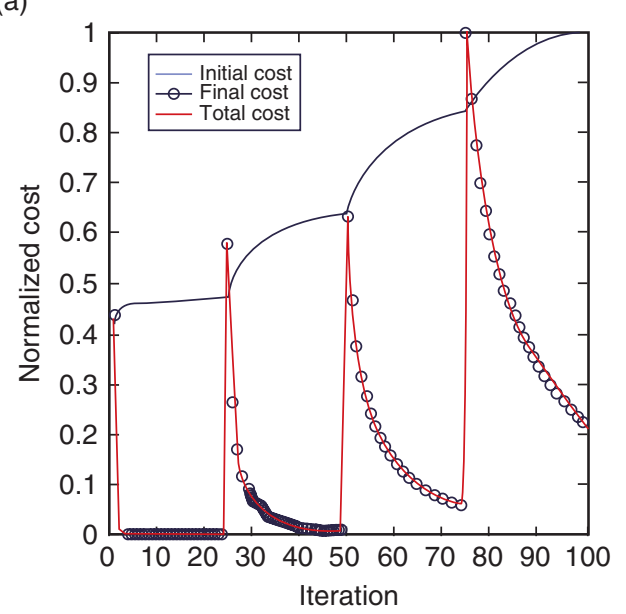

(b)

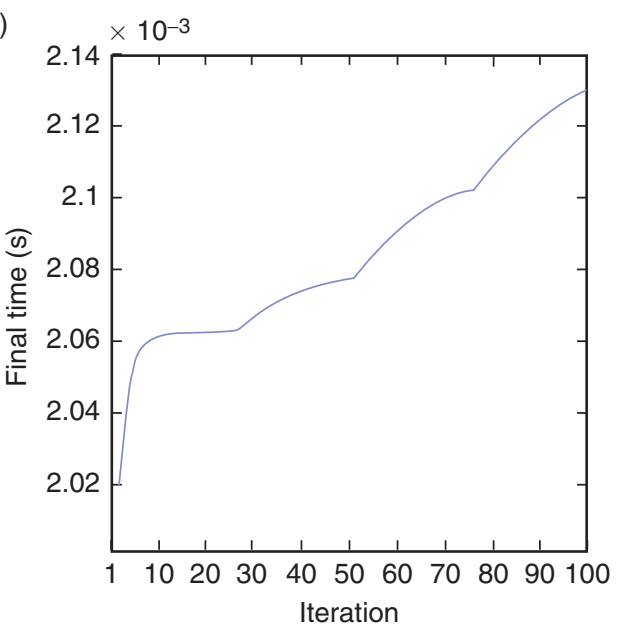

Figure 8. (a) Cost functional $J$ in red and initial penalty $I$ over iterations of solution procedure

(b) Final time, in seconds, vs iteration number for the unconstrained case. The regions of greatest slope are after iterations where the target state has been changed. The slope tending toward zero means that the solution for the optimal final time is converging.

(a)

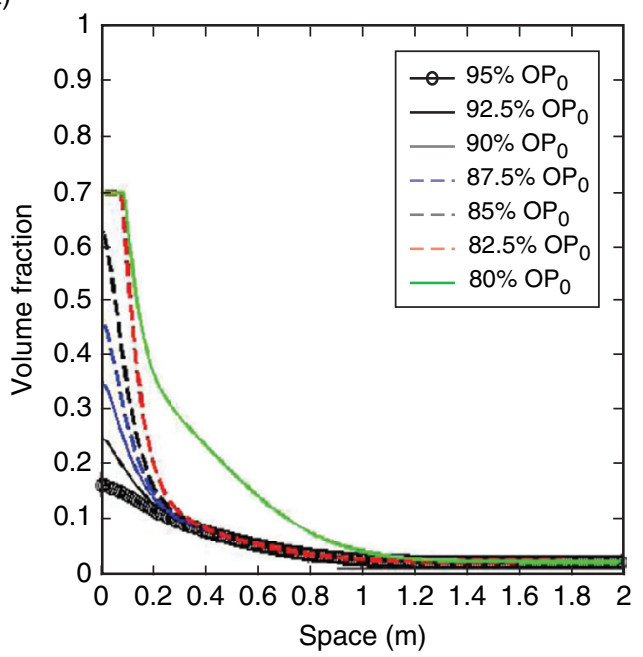

(b)

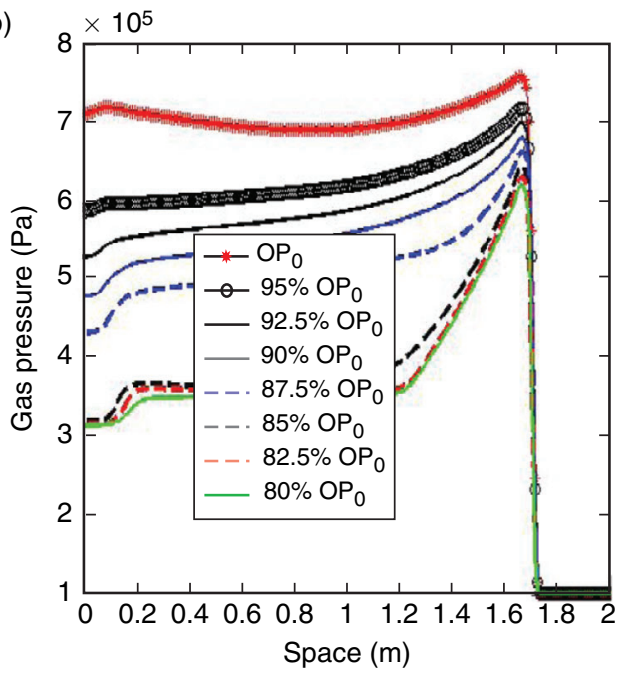

Figure 9. (a) Optimal initial water volume fraction distributions, $\alpha_{l}(x, 0) \leq .7$ (b) The optimal pressure profiles at the final time resulting from the control initial data. 

Application to Ignition Overpressure in Launch Vehicles

A final case was run, this time with an upper bound on the volume fraction of water at $50 \%$. The calculated optimal water distributions are shown in Figure 10.a with the resulting pressure profiles shown in Figure 10.b.

A few more isolated results were obtained in the interest of categorizing the significance of model parameters. Figure 11.a shows the effect of droplet size on overpressure attenuation. Each pressure profile is plotted after two milliseconds of shock propagation from the left boundary toward the right. For a constant amount of water mass, more surface area of the droplets are exposed to the flow when the droplets are smaller. This is verified in eqn (66) for mono-dispersed droplets with diameter $D_{p}$.

$$
\begin{aligned}
& S_{p}=4 \pi\left(\frac{D_{p}}{2}\right)^{2} \cdot N_{p} \\
& N_{p}=\alpha_{l} \frac{\Delta x^{3}}{\frac{4}{3} \pi\left(\frac{D_{p}}{2}\right)^{3}} \\
& \Rightarrow S_{p} \sim \frac{1}{D_{p}}
\end{aligned}
$$

This result agrees with empirical results, [15], that the more droplet surface area exposed, the greater the overpressure attenuation. Since surface area is maximized with infinitely small droplets, initial droplet size isn't an optimizeable parameter. Droplets with a diameter $D_{p}=25 \mu \mathrm{m}$ were used in all of the preceding results since that size is small enough to have a significant effect over two meters, is a reasonable size based on injector atomizer specifications and prevents the droplets from being completely vaporized within the simulation time $T^{*}$. Note that the result in Figure 11.a isolates the effect of droplet size without considering droplet breakup which would presumably diminish the difference in the results to some degree.

Water vapor density was shown to have a drastic effect on results for optimal water distributions. Water vapor density increases by an order of magnitude from ambient pressure to the $8 \mathrm{~atm}$ level behind the shock front of the MP2 data. Therefore, if water vaporizes under high pressures, mass changes phase at a higher steam density and, consequently, the dissipative effect of vaporization is much more significant. The results in Figure 11.b show that more than twice as much water is needed to get 5\% attenuation in pressure depending on the density of the water vapor produced.

(a)

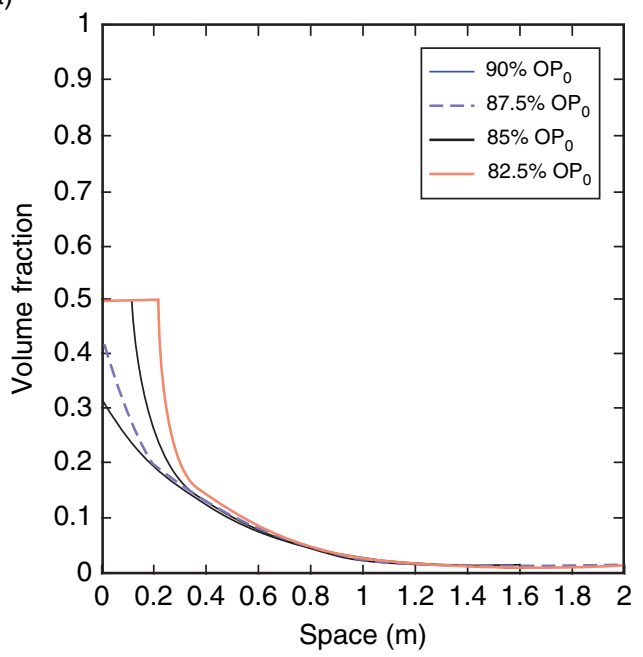

(b)

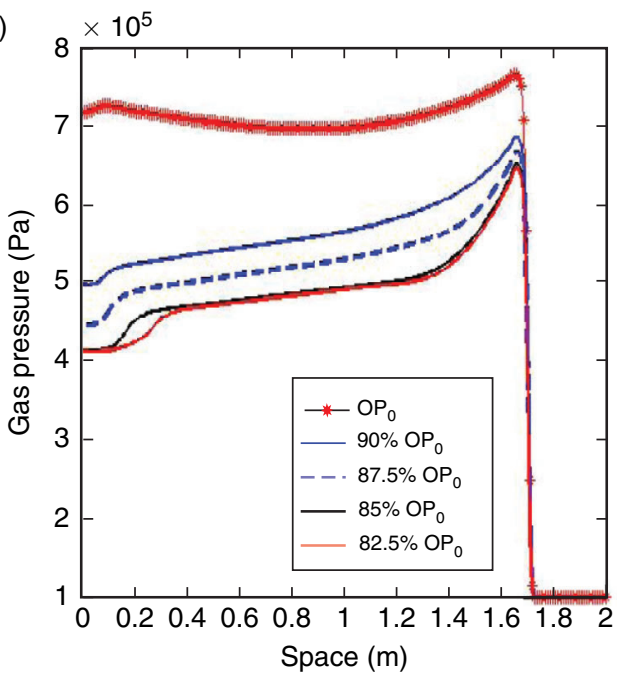

Figure 10. (a) Optimal initial water volume fraction distributions, $\alpha_{l}(x, 0) \leq .5$ (b) Optimal final time pressure profiles resulting from initial data. 
(a)

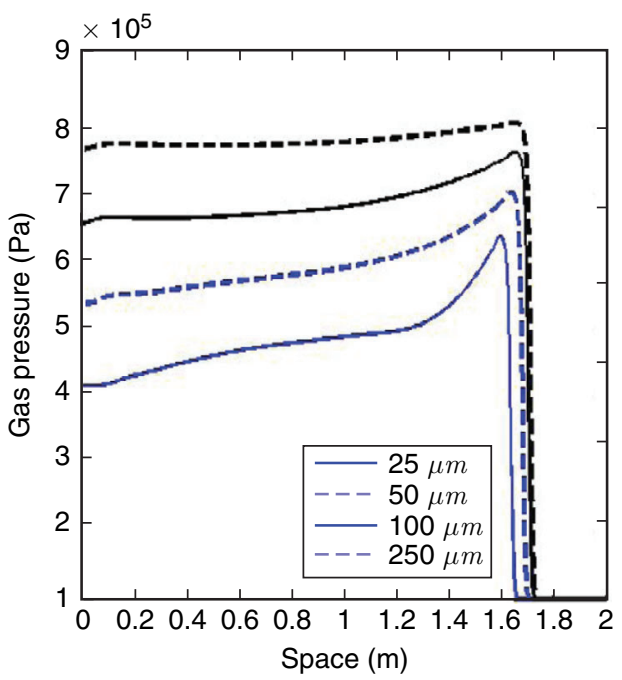

Figure 11. (a) effect of varying droplet size (b)

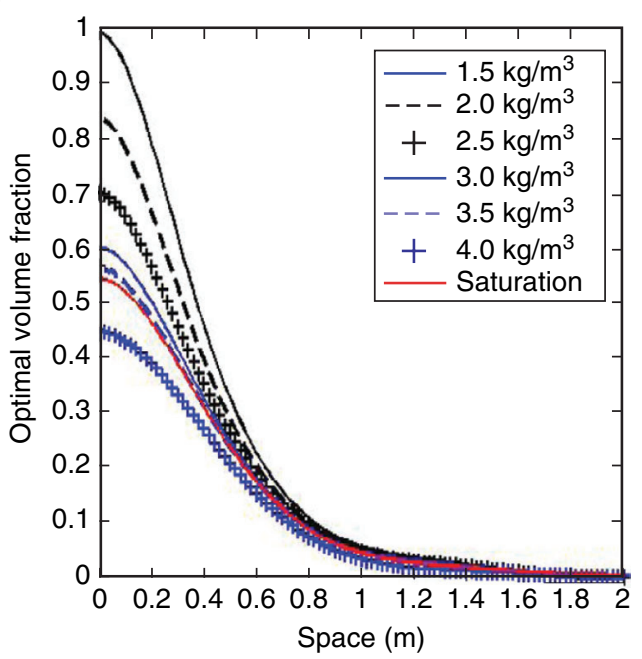

(b) effect of varying water vapor density.

\section{CONCLUSIONS}

A new iterative solution procedure was developed which can calculate optimal distributed control solutions for systems of quasi-linear hyperbolic partial differential equations with free initial and final data and final time. This procedure has been successfully applied to two-phase compressible gas dynamics in one dimension with the goal of diminishing overpressure at the shock front of a blast wave generated by an ignition overpressure. Examples of optimal attenuation to blast waves typically encountered in the launch environment of the Shuttle's SRBs during an ignition are given. Optimal water volume fractions are calculated for increasing levels of attenuation. Cases where the maximum volume fraction of water is restricted to $50 \%$ and $70 \%$ are also presented.

The results give several key insights relevant to implementing water injection systems for blast wave attenuation. Smaller droplets will vaporize quicker since the total surface area exposed to the flow is larger resulting in cooler gas driving a shock with an attenuated jump at the shock front. This would suggest that large regions of space completely filled with water are sub-optimal for blast attenuation since connected streams or ligaments of water expose less surface area to the gas.

The degree of attenuation depends largely on the rate of mass changing phase from liquid water to gaseous water vapor due to forced vaporization $\dot{m}$, which is equal to the product of the water vapor density and the rate of change of the volume occupied by the water droplets. In the high pressure and high temperature region behind the shock front, water vapor density is high and the rate of vaporization is high as well which both contribute to a large value for $\dot{m}$. As the control takes effect, the pressure and temperature of the gas both decrease meaning that the effects are being felt by the shock front at a slower rate. These results show that, in general, stronger shocks can be attenuated more rapidly than weaker shocks via water droplet vaporization.

In a two meter domain using the MP2 data, $76 \% O P_{0}$ was the target with the lowest overpressure for which the solution still converged. The case with the volume fraction of water restricted to a maximum of $\alpha_{l}<70 \%$ converged with the lowest target overpressure of $80 \% O P_{0}$. With the water restricted to below $\alpha_{l}<50 \%$ by volume, the most attenuation achievable with a two meter domain is $82.5 \% O P_{0}$. In a one meter domain using the MP1 data, the lowest target state achievable was $87.5 \%$ $O P_{0}$.

\section{ACKNOWLEDGMENTS}

N. D. Moshman would like to thank Professors Chris Brophy, Frank Giraldo and Wei Kang at the Naval Postgraduate School and Bruce Vu at NASA Kennedy Space Center. In addition, N. D. Moshman thanks the Naval Postgraduate School and the Army Research Office for funding this research. 


\section{REFERENCES}

[1] D. B. Kothe, and W. J. Rider, Comments on Modeling Inter-facial Flows with Volume-of-Fluid Methods, Technical Report LA-UR-3384, Los Alamos National Laboratory, 1995.

[2] R. Caiden, R. P. Fedkiw, and C. Anderson, A Numerical Method for Two-Phase Flow Consisting of Separate Compressible and Incompressible Regions, Journal of Computational Physics, 166, 2001, 1-27.

[3] C. A. Lowe, Two-phase Shock-Tube Problems and Numerical Methods of Solution, Journal of Computational Physics, 204, 2005, 598-632.

[4] R. Saurel, and R. Abgrall, A Multiphase Godunov Method for Compressible Multifluid and Multiphase Flows, Journal of Computational Physics, 150, 1999, 425-467.

[5] S. Lai, and F. S. Laspesa, Ignition Overpressure Measured on STS Lift-Off and Correlation with Subscale Model Tests, JANNAF, 13th Plume Technology Meeting, 1982.

[6] E. J. Walsh, and P. M. Hart, Flight-Measured Lift-off Ignition Overpressure, A Correlation with Sub-scale Model Tests, Martin Marietta Aerospace, AIAA Paper, 1981, 81-2458.

[7] D. Alvord, Ares 1-X Ignition Overpressure T+30 Day Analysis and Results, Jacobs ESTS Group, January 2010, Kennedy Space Center, FL, ESTSG-FY10-00793.

[8] W. G. Reinecke, and G. D. Waldman, A Study of Drop Breakup Behind Strong Shocks with Applications to Flight, AVCO Report, AD-871218, May 1970.

[9] J. Woo Jr., J. H. Jones, and S. H. Guest, A Study of Effects of Water Addition on Supersonic Gas Streams, JANNAF, 13th Plume Technology Meeting, 1982.

[10] C. Crowe, M. Sommerfeld, and Y. Tsuji, Multiphase Flows with Droplets and Particles, CRC Press, New York, 1998.

[11] G. O. Thomas, On the Conditions Required for Explosion Mitigation by Water Sprays, Transactions of the Institution of Chemical Engineers, 78, Part B, September 2000, 339-354.

[12] D. Schwer, and K. Kailasanath, Blast Mitigation by Water Mist (2), Shock Wave Mitigation using Glass Particles and Water Droplets, NRL Memorandum Report, January 21, 2003, 6410-03-8658.

[13] R. Ananth, H. D. Ladouceur, H. D. Willauer, J. P. Farley, and F. W. Williams, Effect of Water Mist on a Confined Blast, Suppression and Detection Research and Applications Technical Working Conference (SUPDET), Orlando, FL., March 11-13, 2008.

[14] H. D. Willauer, R. Ananth, J. P. Farley, G. Back, M. Kennedy, J. O'Connor, and V. M. Gameiro, Blast Mitigation Using Water Mist: Test Series II, NRL Memorandum Report, March 12, 2009, 6180-09-9182.

[15] G. Jourdan, L. Biamino, C. Mariani, C. Blanchot, E. Daniel, J. Massoni, L. Houas, R. Tosello, and D. Praguine, Attenuation of a Shock Wave Passing Through a Cloud of Water Droplets, Shock Waves, 20, No. 4, 2010, 285-296.

[16] H. Ikawa, and F. S. Laspesa, Space Shuttle SRM Ignition Overpressure Prediction Methodology, JANNAF, 13th Plume Technology Meeting, 1982.

[17] C. Kiris, W. Chan, D. Kwak, and J. A. Housman, Time-Accurate Computational Analysis of the Flame Trench, The Fifth International Conference on Computational Fluid Dynamics, Seoul, Korea, July 7-11, 2008.

[18] C. Kiris, J. A. Housman, and D. Kwak, Space-Time Convergence Analysis of a Ignition Overpressure in the Flame Trench, CFD Review 2010, World Scientific.

[19] H. Ikawa, and F. S. Laspersa, Analytical Understanding of WTR Ignition/Duct Overpressure Induced by Space Shuttle Solid Rocket Motor Ignition Transient, AIAA/SAE/ASME $19^{\text {th }}$ Joint Propulsion Conference, Seattle, WA, June 27-29, 1983.

[20] J. Troyes, I. Dubois, V. Borie, and A. Boischot, Multi-phase Reactive Numerical Simulations of a Model Solid Rocket Motor Exhaust Jet, 42 ${ }^{\text {nd }}$ AIAA/ASME/SAI/ASEE Joint Propulsion Conference and Exhibit, Sacramento, CA, July 9-12, 2006.

[21] F. Canabal III, Suppression of the Ignition Overpressure Generated by Launch Vehicles, Ph.D. Dissertation, Mechanical and Aerospace Engineering Department, University of Alabama, Huntsville, 2004. 
[22] F. Canabal III, and A. Frendi, "Study of the Ignition Overpressure Suppression Technique by Water Addition," Journal of Spacecraft and Rockets, 43, No. 4, 2006, 853-865.

[23] N. D. Moshman, G. V. Hobson and S. S. Sritharan, A Method for Optimally Controlling Unsteady Shock Strength in One Dimension, AIAA Journal, accepted for publication July 2012.

[24] R. Saurel, and R. Abgrall, A Simple Method for Compressible Multifluid Flows, SIAM Journal of Scientific Computing 21, No. 3, 2000, 1115-1145.

[25] R. Abgrall, How to Prevent Pressure Oscillations in Multicomponent Flow Calculations: A Quasi Conservative Approach, Journal of Computational Physics, 125, 1996, 150-160.

[26] IAPWS Industrial Formulation 1997 for the Thermodynamic Properties of Water and Steam. (IAPWS-IF97)

[27] B. Texier, Lecture 2: Symmetrizable Systems, RMMC Summer School, Laramie, WY, June 2010.

[28] S. K. Godunov, A Finite difference Method for the Numerical Computation of Discontinuous Solutions of the Equations of Fluid Dynamics, Matematicheskii Sbornik, 47, 1959, 257-393.

[29] B. Van Leer, Toward the Ultimate Conservative Difference Scheme (5) A Second-Order Sequel to Godunov's Method, Journal of Computational Physics, 32, July 1979, 101-136.

[30] T. I. P. Shih, and W. J. Chyu, Approximate Factorization with Source Terms, AIAA Journal Technical Notes, 29, No. 10, 1759-1760.

[31] Gad-el-Hak, M., Flow Control: Passive, Active and Reactive Flow Management, Cambridge University Press, New York, 2000.

[32] Sritharan, S. S. editor. Optimal Control of Viscous Flow, SIAM Books, Philadelphia, PA, 1998.

[33] Gunzburger, M. D. Perspectives in Flow Control and Optimization, SIAM Books, Philadelphia, PA, 2003.

[34] A. Bressan, and A. Marson, A Maximum Principle for Optimally Controlled Systems of Conservations Laws, Rendiconti del Seminario Matematico della Universita di Padova, 94, 1995, 79-94.

[35] Fursikov, A. V., Optimal Control of Distributed Systems: Theory and Applications. American Mathematical Society, Providence, Rhode Island, 2000.

[36] A. Bressan, and A. Marson, A Variational Calculus for Discontinuous Solutions to Conservation Laws, Communications on Partial Differential Equations, 20, 1995, 1491-1552.

[37] S. Ulbrich, A Sensitivity and Adjoint Calculus for Discontinuous Solutions of Hyperbolic Conservation Laws with Source Terms, SIAM Journal of Control and Optimization, 41, 2002, 740-783.

[38] S. Ulbrich, Adjoint-Based Derivative Computations for the Optimal Control of Discontinuous Solution of Hyperbolic Conservation Laws, Systems and Control Letters, 3, 2003, 309-323.

[39] Moshman, N. D. 2011 Optimal Control of Unsteady Shock Wave Attenuation in Single-and Two-Phase Flow with Application to Ignition Overpressure in Launch Vehicles. Ph.D. Dissertation, Mechanical and Astronautical Engineering Department, Naval Postgraduate School, Monterey, CA.

[40] M. B. Giles, and N. A. Pierce, Adjoint Equations in CFD; Duality, Boundary Conditions and Solution Behavior, AIAA-97-1850, A97-32424, pp. 182-198.

[41] R. Giering, and T. Kaminski, Recipes for Adjoint Code Construction, ACM Transactions on Mathematical Software, 24, No. 4, December 1998, 437-474.

[42] C. Bushens, and H. Maurer, SQP-Methods for Solving Optimal Control Problems with Control and State Constraints; Adjoint Variables, Sensitivity Analysis and Real-Time Control, Journal of Computational and Applied Mathematics, 120, 2000, 85-108.

[43] E. E. Prudencio, R. Byrd, and X. C. Cai, Parallel Full Space SQP Lagrange-Newton-KrylovSchwarz Algorithms for PDE-Constrained Optimization Problems, SIAM Journal of Scientific Computation 27, No. 4, 2006, 1305-1328.

[44] M. K. Banda, and M. Herty, Adjoint IMEX-based Schemes for Control Problems Governed by Hyperbolic Conservation Laws, Computational Optimization and Applications, published online October 14, 2010, Springer, DOI: 10.1007/s10589-010-9362-2.

[45] A. K. Alekseev, and I. M. Navon, On Adjoint Variables for Discontinuous Flow, Submitted for 
publication in Systems and Control Letters, 2002.

[46] G. Sutton, and O. Biblarz, Solid Propellant Rocket Fundamentals, Rocket Propulsion Elements, 8th Ed., John Wiley and Sons. Hoboken, New Jersey, 2010, 437-438.

[47] Cequel, Chemical Equilibrium in Excel, (C2003, Software and Engineering Associates, Inc.

[48] CFD-FASTRAN User's Manual, version 2002, CFD Research Corporation, Huntsville, AL, 2002. 